OPEN ACCESS

Edited by:

Sei Saitoh,

Fujita Health University, Japan

Reviewed by:

Toshitaka Oohashi,

Okayama University, Japan

Hiroshi Kitagawa,

Kobe Pharmaceutical University,

Japan

Yuki Hirota,

Keio University, Japan

*Correspondence:

Eri Arikawa-Hirasawa ehirasaw@juntendo.ac.jp

Received: 25 August 2021 Accepted: 14 September 2021 Published: 04 October 2021

Citation:

Kerever $A$ and

Arikawa-Hirasawa E (2021) Optimal Extracellular Matrix Niches

for Neurogenesis: Identifying

Glycosaminoglycan Chain Composition in the Subventricular

Neurogenic Zone.

Front. Neuroanat. 15:764458. doi: 10.3389/fnana.2021.764458

\section{Optimal Extracellular Matrix Niches for Neurogenesis: Identifying Glycosaminoglycan Chain Composition in the Subventricular Neurogenic Zone}

\author{
Aurelien Kerever ${ }^{1}$ and Eri Arikawa-Hirasawa ${ }^{1,2 *}$ \\ ${ }^{1}$ Research Institute for Diseases of Old Age, Juntendo University Graduate School of Medicine, Tokyo, Japan, ${ }^{2}$ Department \\ of Neurology, Juntendo University School of Medicine, Tokyo, Japan
}

In the adult mammalian brain, new neurons are generated in a restricted region called the neurogenic niche, which refers to the specific regulatory microenvironment of neural stem cells (NSCs). Among the constituents of neurogenic niches, the extracellular matrix (ECM) has emerged as a key player in NSC maintenance, proliferation, and differentiation. In particular, heparan sulfate (HS) proteoglycans are capable of regulating various growth factor signaling pathways that influence neurogenesis. In this review, we summarize our current understanding of the ECM niche in the adult subventricular zone (SVZ), with a special focus on basement membrane (BM)-like structures called fractones, and discuss how fractones, particularly their composition of glycosaminoglycans (GAGs), may influence neurogenesis.

\footnotetext{
Keywords: fractone, heparan sulfate chains, neurogenesis, subventricular zones, extracellular matrix, growth factors, glycosaminoglycans
}

\section{INTRODUCTION}

In the adult mouse brain, neurogenesis occurs continuously in the subventricular zone (SVZ) of the lateral ventricle (Altman, 1963; Doetsch et al., 1997) and the subgranular zone of the hippocampal dentate gyrus (Seki and Arai, 1993; Eriksson et al., 1998). In the adult SVZ, type B stem cells give rise to type C transit-amplifying cells, which, in turn, produce type A neuroblasts (Doetsch, 2003). These neuroblasts migrate toward the olfactory bulb along the rostral migratory stream, where they mature into GABAergic interneurons (Lois and Alvarez-Buylla, 1994; Alvarez-Buylla et al., 2002; Kriegstein and Alvarez-Buylla, 2009). The complex microenvironment that supports this series of events is commonly referred to as the neurogenic niche. This niche consists of various cell types that surround neural stem cells (NSCs), such as neural stem and progenitor cells, ependymocytes, mature and immature neurons, and astrocytes, as well as the vasculature. The extracellular matrix (ECM) is another critical component of this niche. Notably, NSCs have been shown to contact the basement membrane (BM) of the vasculature at sites lacking astrocyte endfeet and 
pericyte coverage (Tavazoie et al., 2008). In addition, vascular BM NSCs also contact a local ECM structure called fractones (Figures 1A,B).

\section{FRACTONE: EXTRACELLULAR MATRIX NICHE IN THE SUBVENTRICULAR ZONE}

Fractones are extravascular ECM structures that are localized along the ventricular wall. These structures were initially observed through laminin immunostaining as small punctate structures of $2-5 \mu \mathrm{m}$, located behind the ependyma (arrow, Figures 1A-C). However, transmission electron microscopy revealed that fractones are electron-dense structures with branched morphology that allow them to contact numerous surrounding cells, including ependymocytes, astrocytes, NSC, and progenitor cells (Mercier et al., 2002, 2003). The ependymal wall contains interstitial clefts that allow the diffusion of signaling molecules from the cerebrospinal fluid (Brightman, 2002). Fractones are located at the end of these narrow channels and are ideally placed to receive growth factors and cytokines produced by the choroid plexus (Kerever et al., 2007; Mercier, 2016). Fractones first appear around postnatal day 5 and are composed of a ubiquitous BM component (Kerever et al., 2007; Nascimento et al., 2018; Sato et al., 2019). The presence of fractones rich in $\mathrm{BM}$ protein may participate in increasing the tissue stiffness of the neurogenic niche (Kjell et al., 2020). Recent studies have proposed ependymocytes (Nascimento et al., 2018) and GFAP-expressing cells (Sato et al., 2019) as cells that produce fractones. This suggests that the formation of fractones results from the contribution of various cells in the niche.

While fractone protein composition closely resembles that of the vascular $\mathrm{BM}$, the fractone heparan sulfate (HS) composition is unique. HS chains belong to the Glycosaminoglycans (GAGs) family. GAGs are long, unbranched, hydrophilic, highly charged chains composed of repeating disaccharide units that can be classified into four groups based on their core disaccharide structure: keratan, hyaluronan, chondroitin sulfate/dermatan sulfate, and HS. Only HS chains can be found in vascular BM and fractones. N-sulfated HS chains recognized by 10E4 epitope immunoreactivity suggests that fractones HS present higher levels of sulfation than HS from the vascular BM (Figure 1C; Kerever et al., 2007).

Fractones are composed of ubiquitous BM components. Collagen type IV, the most abundant component of the $\mathrm{BM}$, forms a network-like structure and is linked to a network of laminins with the help of nidogen/entactin (Pozzi et al., 2017). In addition, fractones contain two major types of heparan sulfate proteoglycans (HSPGs), perlecan and agrin (Figure 1D). The other main member of the BM type of HSPG, collagen XVIII, remains undetected in either fractones or vascular BM in the SVZ (Kerever et al., 2007).

Fractones may play various roles in the neurogenic niche through laminin-integrin interactions (Shen et al., 2008;
Nascimento et al., 2018; Sato et al., 2019) by regulating heparinbinding ligand availability (Kerever et al., 2007) in the niche and promoting growth factor signaling (Douet et al., 2012; Kerever et al., 2014; Mercier and Douet, 2014). NSC interaction with laminin through $\alpha 6 \beta 1$ integrin expressed on its cell surface is essential to maintain NSC quiescence (Shen et al., 2008). Laminins are heterotrimeric glycoproteins composed of $1 \alpha$, $1 \beta$, and $1 \gamma$ chains. The $\beta$ and $\gamma$ chains coil around the $\alpha$ chain to form a cross-like structure with three short and one long arms. Short arms are responsible for self-polymerization and interactions with other BM molecules (Hohenester and Yurchenco, 2013). The longer arm most notably interacts with integrin and dystroglycan on the cell surface, leading to cytoskeleton rearrangement and impacting cell behavior. Various laminin isoforms can be found in fractones and the vascular BM in the neurogenic niche. While laminin $\alpha 1$ and $\gamma_{2}$ are absent from both fractones and the vascular BM, laminin $\alpha 5, \beta 1 / 2$, and $\gamma 1$ are present in both. In addition, laminin $\alpha 2$ and $\alpha 4$ are present only in the vascular BM, but $\alpha 3$ is only present in fractones (Kerever et al., 2007; Nascimento et al., 2018; Sato et al., 2019). Other glycoproteins such as secreted modular calcium-binding protein 1 and 2 (SMOC1/2) and the laminin-related molecule netrin 4, which shares homology with the N-terminal portion of laminin $\beta 1$, (Sun et al., 2011) have also been detected in fractones (Sato et al., 2019).

The capacity of fractones to specifically capture heparinbinding growth factors from the extracellular milieu highlights a critical role for its HSPG perlecan and agrin. Perlecan, also referred to as HSPG 2, is a major BM type of HSPG, and deficiency of perlecan causes perinatal lethality in mice and humans (Arikawa-Hirasawa et al., 1999, 2001). The core protein is composed of five distinct domains and interacts with a variety of molecules from the ECM (laminin, nidogen, collagen IV; Figure 1D). Through its protein core and its HS chains, perlecan is involved in numerous biological processes, including embryonic development, tissue homeostasis, and pathology (Gubbiotti et al., 2017; Yu et al., 2017). In the neurogenic niche, perlecan is present in both vascular BM and fractones, and we previously reported that the presence of perlecan in fractones through its HS chains promoted FGF-2 stimulation of neurogenesis (Kerever et al., 2014).

Agrin is another major HSPG component of the BM, and plays a critical role in the hematopoietic stem cell niche (Mazzon et al., 2011; Pozzi et al., 2017). Agrin is also a key component of the microenvironment that regulates synapse differentiation at the neuromuscular junction (Gautam et al., 1996) and in neurons of the hippocampus (Böse et al., 2000), as well as in newborn neurons of the olfactory bulb (Burk et al., 2012). In the SVZ, agrin is found in both the vascular BM and fractones (Kerever et al., 2014).

In addition to the cell/ECM interaction that plays a role in maintaining NSC quiescence through laminin/integrin signaling, fractones also play a role in regulating growth factor signaling. Both perlecan and agrin bear HS chains, and might therefore 


\section{A}

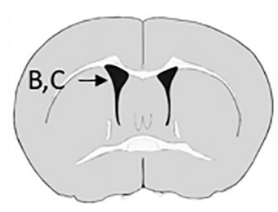

B SVZ neurogenic niche

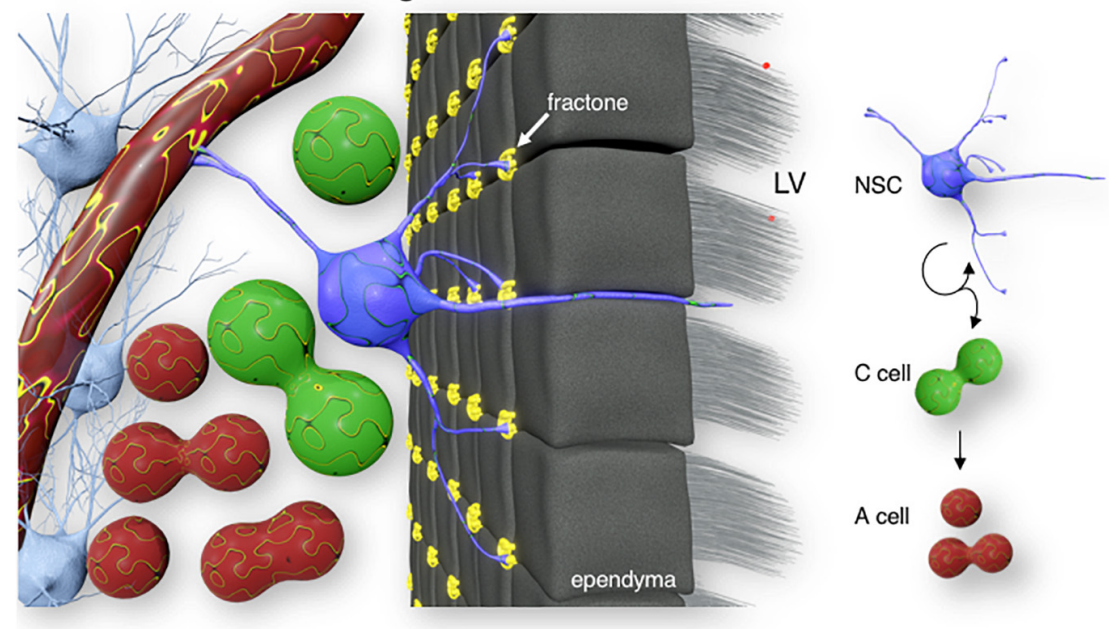

C

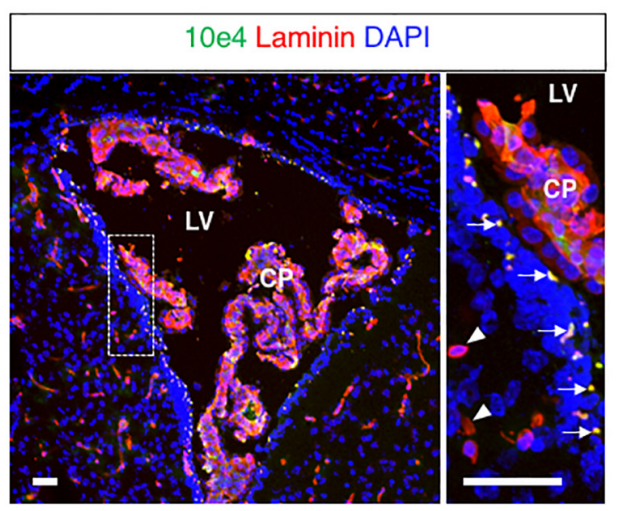

D

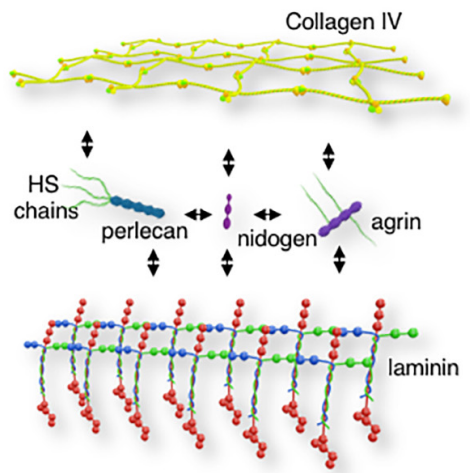

FIGURE 1 | Fractone: extracellular matrix (ECM) niche in the SVZ. (A) A schematic of a mouse brain coronal section at bregma $0.1 \mathrm{~mm}$, with an inset displaying the localization of the lateral ventricle shown in panels (B,C). (B) 3D rendering of the SVZ neurogenic niche showing a single NSC (blue) contacting the ventricle lumen, a capillary, and numerous fractones (yellow). (C) Confocal image of the lateral ventricle displaying laminin (red) and N-Sulfated HS epitope 10E4 (green) immunoreactivity in the SVZ. Arrows indicate the fractones that are immunoreactive for both laminin and N-sulfated HS epitope 10E4. Arrowheads indicate laminin-immunoreactive SVZ capillaries. Scale bar: $50 \mu \mathrm{m}$. (D) A schematic of major fractones ECM component.

contribute to the regulation of heparin-binding growth factor signaling in the SVZ.

\section{HEPARAN SULFATE CHAIN STRUCTURES REGULATE GROWTH FACTOR SIGNALING}

The building blocks of HS are glucuronic acid (GlcA) and $\mathrm{N}$-acetylglucosamine (GlcNAc). They are alternatively transferred to a linker composed of one xylose residue, two galactose residues, and one GlcA residue. This polysaccharide subsequently undergoes extensive modification in the Golgi apparatus, which is catalyzed by a series of enzymes. First, $\mathrm{N}$-deacetylase/N-sulfotransferase (NDST) acts on a subset of GlcNAc residues to produce N-sulfated glucosamine (GlcNS). This enzyme also generates a small number of $\mathrm{N}$-unsubstituted glucosamine residues due to incomplete $\mathrm{N}$-sulfation. Then, a glucuronyl C5-epimerase (GLCE) acts on the GlcA residue to create Iduronic acid (IdoA), followed by the action of HS 2-sulfotransferases that catalyze the transfer of a sulfate to the C2-position of uronic acid residues. Subsequently, HS 6-sulfotransferases (HS6ST) catalyze the transfer of sulfate onto the C6 position of the glucosamine residue in HS. 
Finally, HS 3-sulfotransferases (HS3ST) can transfer sulfate to the $3-\mathrm{OH}$ position of the glucosamine residues of $\mathrm{HS}$ (Sugahara and Kitagawa, 2002; Kreuger and Kjellén, 2012). Upon release into the extracellular space, secreted endosulfatase (Sulf1 and Sulf2) can catalyze the removal of a subset of $6 \mathrm{O}$ sulfated group from the HS chains. The modification reactions in heparan sulfate biosynthesis occur in clusters along the polysaccharide, resulting in a highly sulfated region ( $S$ domain) separated by regions devoid of sulfate (NA domain) (Figure 2). Together, these steps contribute to the formation of a sulfated polysaccharide with tremendous chemical heterogeneity, allowing HS chains to specifically interact with a wide range of molecules (Annaval et al., 2020).

Binding of HS to a ligand may impact signaling in various ways, thereby key stem cell function (Ravikumar et al., 2020). In addition, extracellular modification of HS sulfation by endosulfatase can actively modulate growth factor signaling.

\section{0-Sulfation}

6O-sulfation is regulated both during biosynthesis by HS6ST enzymes that add a sulfate group to the glucosamine residue and post synthetically in the extracellular space by endosulfatase that can remove a subset of $6 \mathrm{O}$ sulfate group (Annaval et al., 2020). Level of 6O-sulfation than has a great impact on numerous cell-signaling pathways.

For instance, 6O-sulfated HS bind Wnt with high affinity and thereby negatively regulate Wnt activity by preventing access to its receptor (Frizzled) on the cell surface. Removal of 6Osulfation has been shown to reduce Wnt affinity for HS, allowing the formation of an HS/Wnt/Fz complex (Ai et al., 2003). Such regulation has great implications in the neurogenic niche, as Wnt has been shown to play a critical role in regulating the fate of NSCs (Hirota et al., 2016; Kriska et al., 2016).

A similar mechanism has been reported for bone morphogenetic protein (BMP). The BMP antagonist Noggin binds to highly sulfated HS, but Sulf activity leads to the release of Noggin and restores BMP signaling (Viviano et al., 2004). Noggin expressed by ependymocytes has been shown to promotes neurogenesis by blocking BMP signaling (Lim et al., 2000). BMP4 and BMP7 have been shown to specifically bind to Fractones HS and inhibit cell proliferation in the neurogenic niche (Douet et al., 2012; Mercier and Douet, 2014).

Another strategy involves HS acting as a coreceptor with FGF2 as a prime example. HS is necessary for the formation of the ternary complex of basic fibroblast growth factor (FGF-2), FGF receptor (FGFR), and HS (Mohammadi et al., 2005).

$6 \mathrm{O}$-sulfation is not required for FGF2 binding to $\mathrm{HS}$, but it is necessary for the formation of the ternary complex and subsequent cell signaling (Guimond et al., 1993; Pye et al., 1998). Therefore, Sulf provides the possibility to finely tune FGF-2 activity by converting a coreceptor type of HS into one that stores FGF-2 and prevents downstream signaling. In the neurogenic niche, FGF-2 specifically binds to fractones HS, and this interaction is necessary for FGF-2 stimulation of cell proliferation (Kerever et al., 2007; Douet et al., 2013).

Regulation of HS capacity to act as a co-receptor by Sulf has also been reported for numerous other growth factors, including

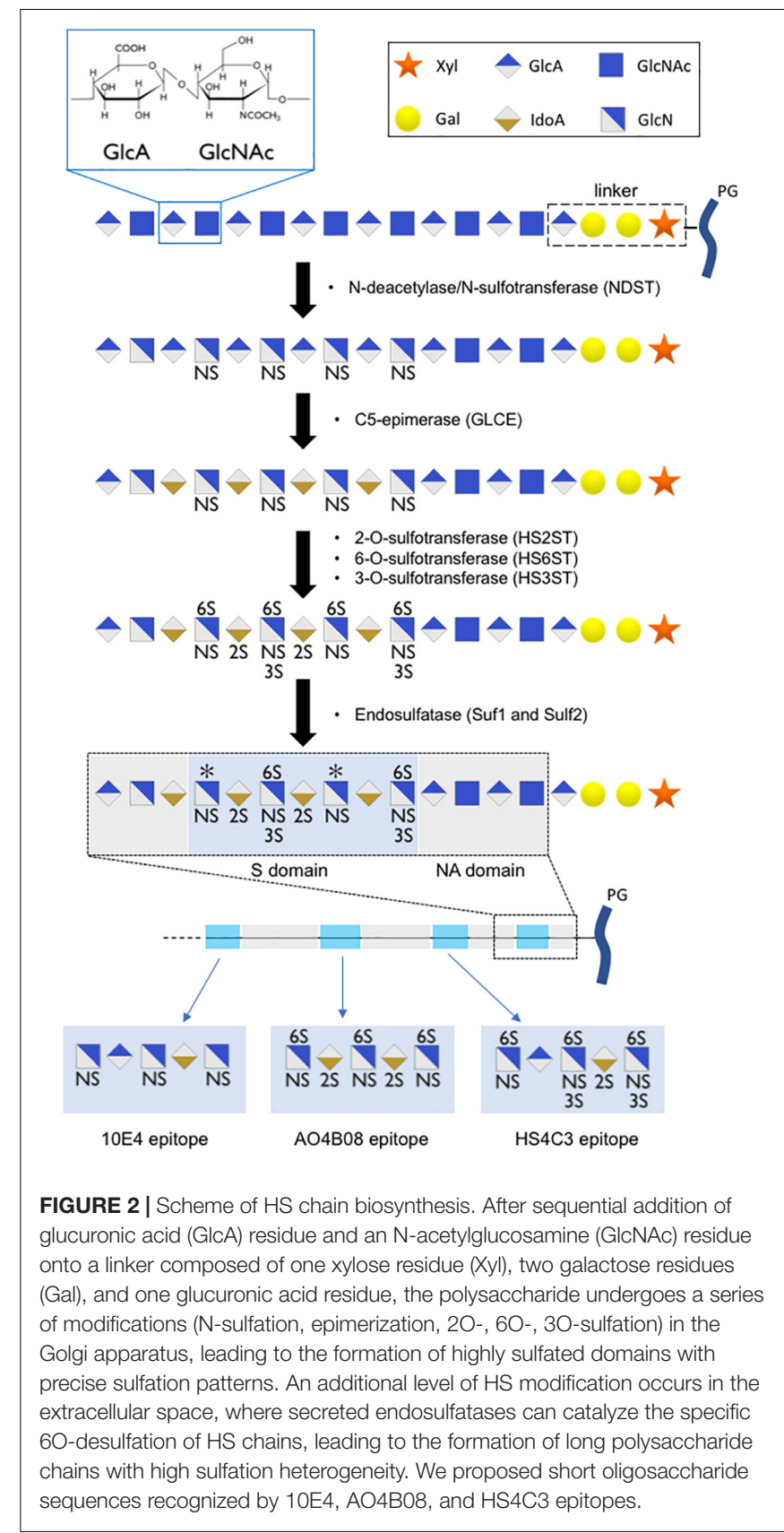

amphiregulin (Narita et al., 2007), hepatocyte growth factor (HGF; Lai et al., 2004), heparin-binding epidermal growth factorlike growth factor (HB-EGF; Lai et al., 2003), FGF-1, stromal cell-derived factor-1 (SDF-1), and vascular endothelial growth factor (VEGF; Uchimura et al., 2006). These results demonstrate that the regulation of $6 \mathrm{O}$-sulfation alone can impact numerous signaling pathways. 6O-sulfation was detected using the antiHS phage display antibody AO4B08. This antibody recognizes a short oligosaccharide sequence that includes N-sulfation, C5epimerization, 2O-sulfation, and high levels of 6O-sulfation (Dennissen et al., 2002; ten Dam et al., 2003; Kurup et al., 2007). A short oligosaccharide sequence recognized by AO4B08 
antibody is presented in Figure 2. We recently reported that fractones display high AO4B08 immunoreactivity (Kerever et al., 2021). Vascular BM in the SVZ displayed weaker AO4B08 immunoreactivity. In addition, it is noteworthy that AO4B08 immunoreactivity in fractones is heterogeneous. Thus, fractones on the dorso-lateral side of the ventricle displayed stronger AO4B08 immunoreactivity than fractones on the ventral side of the ventricle (Kerever et al., 2021).

\section{0-Sulfation}

HS 3-sulfotransferases enzymes that catalyze the transfer of a sulfate on the 30-position of the glucosamine residue have been shown to display different subcellular localizations. In particular, HS3ST2 can be found in the plasma membrane, while HS3ST3B resides in the Golgi apparatus (Delos et al., 2018). This difference in localization may result in the formation of distinct sulfation motifs. 3O-sulfation has been reported to modulate ligand binding (Chopra et al., 2021). Detection of 3O-sulfation can be performed using the anti-HS phage display antibody HS4C3 (van Kuppevelt et al., 1998; ten Dam et al., 2006; Hirano et al., 2012). HS4C3 recognizes a short oligosaccharide sequence that includes $\mathrm{N}$-sulfation, C5-epimerization, 2O-sulfation, 6Osulfation, and 30-sulfation. A short oligosaccharide sequence recognized by HS4C3 antibody is presented in Figure 2. We recently reported that fractones displayed strong HS4C3 immunoreactivity (Kerever et al., 2021).

\section{AGING OF THE NEUROGENIC NICHE}

Fractones have been shown to be altered under some pathological conditions. In the SVZ of BTBR $t+t f / J$ mice, a mouse model of autism spectrum disorder, fractones were reported to be drastically reduced in size and number (Mercier et al., 2011, 2012). In contrast, the size of fractones has been reported to increase following long-term hydrocephalus in adult mice (Campos-Ordoñez et al., 2014). In addition, we previously reported that the structure and composition of fractones were altered in aged mouse SVZ (Kerever et al., 2015; Yamada et al., 2017). With aging, neurogenesis declines, (Maslov et al., 2004) and the neurogenic niche undergoes structural and functional remodeling (Luo et al., 2006; Rojas-Vázquez et al., 2021). Ependymal cells present altered morphology, their number decreases while the number of astrocytes interposed between ependymocytes increases (Luo et al., 2008; Capilla-Gonzalez et al., 2014). Additionally, the blood brain barrier in SVZ capillaries is compromised, leading circulating pro-inflammatory molecules to potentially affect the niche (Obermeier et al., 2013; Segarra et al., 2021).

Fractone size gradually increases with age (Kerever et al., 2015; Nascimento et al., 2018). In addition, the HS composition of

\section{REFERENCES}

Ai, X., Do, A. T., Lozynska, O., Kusche-Gullberg, M., Lindahl, U., and Emerson, C. P. (2003). QSulf1 remodels the 6-O sulfation states of cell surface heparan fractones was also modified in the aged SVZ. We previously reported on disaccharide analysis of the young and aged SVZ that total 6O-sulfation decreased in the aged SVZ. This loss of 6O-sulfation was accompanied by impaired FGF-2 signaling (Yamada et al., 2017). The aged fractones also displayed reduced immunoreactivity for the $\mathrm{N}$-sulfated epitope 10E4 (Kerever et al., 2015) and reduced AO4B08 immunoreactivity (Kerever et al., 2021). This suggests dramatic changes in the sulfation of fractones HS, and these changes may affect growth activity and participate in the age-related decline of neurogenesis.

\section{CONCLUSION}

As we have described, minute modification of sulfation along the HS chains leads to dramatic changes in HS regulation of growth factor signaling. Therefore, it is critical to identify strategies to reveal precise HS sequences. The biochemical approach, which consists of breaking apart the HS chains into disaccharide units, is helpful to obtain broad information pertaining to HS composition, but it fails to elucidate the actual organization within the HS chain and cannot provide information on HS heterogeneity in the microenvironment. As we previously reported in the context of the SVZ, immunoreactivity for 10E4 shows that HS displays dramatically different sulfation signatures in the vascular BM than in fractones. The development of specific anti-HS antibodies is a great tool for deciphering the HS code (van Kuppevelt et al., 1998; Dennissen et al., 2002; Thompson et al., 2009), and studying the influence of local changes in HS composition on cell signaling in health and pathological conditions.

\section{AUTHOR CONTRIBUTIONS}

$\mathrm{AK}$ and EA-H conceptualized the manuscript. Both authors drafted the manuscript and have reviewed and agreed with the publication of the manuscript.

\section{FUNDING}

This work was supported by the Grants-in-Aid for Scientific Research from JSPS KAKENHI (20K08089 to AK and $18 \mathrm{~K} 07535$ to EA-H).

\section{ACKNOWLEDGMENTS}

We thank Hiroyuki Hioki for his critical reading of the manuscript.

sulfate proteoglycans to promote Wnt signaling. J. Cell Biol. 162, 341-351. doi: $10.1083 /$ jcb.200212083

Altman, J. (1963). Autoradiographic investigation of cell proliferation in the brains of rats and cats. Anatom. Record 145, 573-591. doi: 10.1002/ar.1091450409 
Alvarez-Buylla, A., Seri, B., and Doetsch, F. (2002). Identification of neural stem cells in the adult vertebrate brain. Brain Res. Bull. 57, 751-758. doi: 10.1016/ S0361-9230(01)00770-5

Annaval, T., Wild, R., Crétinon, Y., Sadir, R., Vivès, R. R., and Lortat-Jacob, H. (2020). Heparan sulfate proteoglycans biosynthesis and post synthesis mechanisms combine few enzymes and few core proteins to generate extensive structural and functional diversity. Molecules 25:4215. doi: 10.3390/ molecules 25184215

Arikawa-Hirasawa, E., Watanabe, H., Takami, H., Hassell, J. R., and Yamada, Y. (1999). Perlecan is essential for cartilage and cephalic development. Nat. Genet. 23, 354-358. doi: 10.1038/15537

Arikawa-Hirasawa, E., Wilcox, W. R., Le, A. H., Silverman, N., Govindraj, P., Hassell, J. R., et al. (2001). Dyssegmental dysplasia, Silverman-Handmaker type, is caused by functional null mutations of the perlecan gene. Nat, Genet. 27, 431-434. doi: 10.1038/86941

Böse, C. M., Qiu, D., Bergamaschi, A., Gravante, B., Bossi, M., Villa, A., et al. (2000). Agrin controls synaptic differentiation in hippocampal neurons. J. Neurosci. 20, 9086-9095. doi: 10.1523/jneurosci.20-24-09086.2000

Brightman, M. W. (2002). The brain's interstitial clefts and their glial walls. J. Neurocytol. 31, 595-603. doi: 10.1023/A:1025783326667

Burk, K., Desoeuvre, A., Boutin, C., Smith, M. A., Kroger, S., Bosio, A., et al. (2012). Agrin-signaling is necessary for the integration of newly generated neurons in the adult olfactory bulb. J. Neurosci. 32, 3759-3764. doi: 10.1523/JNEUROSCI. 4906-11.2012

Campos-Ordoñez, T., Herranz-Pérez, V., Chaichana, K. L., Rincon-Torroella, J., Rigamonti, D., García-Verdugo, J. M., et al. (2014). Long-term hydrocephalus alters the cytoarchitecture of the adult subventricular zone. Exp. Neurol. 261, 236-244. doi: 10.1016/j.expneurol.2014.05.011

Capilla-Gonzalez, V., Cebrian-Silla, A., Guerrero-Cazares, H., Garcia-Verdugo, J. M., and Quiñones-Hinojosa, A. (2014). Age-related changes in astrocytic and ependymal cells of the subventricular zone. Glia 62, 790-803. doi: 10.1002/glia. 22642

Chopra, P., Joshi, A., Wu, J., Lu, W., Yadavalli, T., Wolfert, M. A., et al. (2021). The 3-O-sulfation of heparan sulfate modulates protein binding and lyase degradation. Proc. Natl. Acad. Sci. U.S.A. 118, 1-12. doi: 10.1073/PNAS. 2012935118

Delos, M., Foulquier, F., Hellec, C., Vicogne, D., Fifre, A., Carpentier, M., et al. (2018). Heparan sulfate 3-O-sulfotransferase 2 (HS3ST2) displays an unexpected subcellular localization in the plasma membrane. Biochim. Biophys. Acta Gen. Sub. 1862, 1644-1655. doi: 10.1016/j.bbagen.2018.04.013

Dennissen, M. A. B. A., Jenniskens, G. J., Pieffers, M., Versteeg, E. M. M., Petitou, M., Veerkamp, J. H., et al. (2002). Large, tissue-regulated domain diversity of heparan sulfates demonstrated by phage display antibodies. J. Biol. Chem. 277, 10982-10986. doi: 10.1074/jbc.M104852200

Doetsch, F. (2003). The glial identity of neural stem cells. Nat. Neurosci. 6, 1127-1134. doi: 10.1038/nn1144

Doetsch, F., Garcì-Verdugo, J. M., and Alvarez-Buylla, A. (1997). Cellular composition and three-dimensional organization of the subventricular germinal zone in the adult mammalian brain. J. Neurosci. 17, 5046-5061. doi: 10.1523/JNEUROSCI.17-13-05046.1997

Douet, V., Arikawa-Hirasawa, E., and Mercier, F. (2012). Fractone-heparan sulfates mediate BMP-7 inhibition of cell proliferation in the adult subventricular zone. Neurosci. Lett. 528, 120-125. doi: 10.1016/j.neulet.2012.08.077

Douet, V., Kerever, A., Arikawa-Hirasawa, E., and Mercier, F. (2013). Fractoneheparan sulphates mediate FGF-2 stimulation of cell proliferation in the adult subventricular zone. Cell Prolif. 46, 137-145. doi: 10.1111/cpr.12023

Eriksson, P. S., Perfilieva, E., Björk-Eriksson, T., Alborn, A.-M., Nordborg, C., Peterson, D. A., et al. (1998). Neurogenesis in the adult human hippocampus. Nat. Med. 4, 1313-1317. doi: 10.1038/3305

Gautam, M., Noakes, P. G., Moscoso, L., Rupp, F., Scheller, R. H., Merlie, J. P., et al. (1996). Defective neuromuscular synaptogenesis in agrin-deficient mutant mice. Cell 85, 525-535. doi: 10.1016/s0092-8674(00)81253-2

Gubbiotti, M. A., Neill, T., and Iozzo, R. V. (2017). A current view of perlecan in physiology and pathology: a mosaic of functions. Matrix Biol. 57-58, 285-298. doi: 10.1016/j.matbio.2016.09.003

Guimond, S., Maccarana, M., Olwin, B. B., Lindahl, U., and Rapraeger, A. C. (1993). Activating and inhibitory heparin sequences for FGF-2 (basic FGF). Distinct requirements for FGF-1, FGF-2, and FGF-4. J. Biol. Chem. 268, 23906-23914. doi: 10.1016/s0021-9258(20)80471-2

Hirano, K., Sasaki, N., Ichimiya, T., Miura, T., van Kuppevelt, T. H., and Nishihara, S. (2012). 3-O-sulfated heparan sulfate recognized by the antibody HS4C3 contribute to the differentiation of mouse embryonic stem cells via fas signaling. PLoS One 7:e0043440. doi: 10.1371/journal.pone.0043440

Hirota, Y., Sawada, M., Huang, S.-H., Ogino, T., Ohata, S., Kubo, A., et al. (2016). Roles of Wnt signaling in the neurogenic niche of the adult mouse ventricularsubventricular zone. Neuroch. Res. 41, 222-230. doi: 10.1007/s11064-015-1 766-z

Hohenester, E., and Yurchenco, P. D. (2013). Laminins in basement membrane assembly. Cell Adhesion Migr. 7, 56-63. doi: 10.4161/cam.21831

Kerever, A., Mercier, F., Nonaka, R., de Vega, S., Oda, Y., Zalc, B., et al. (2014). Perlecan is required for FGF-2 signaling in the neural stem cell niche. Stem Cell Res. 12, 492-505. doi: 10.1016/j.scr.2013.12.009

Kerever, A., Schnack, J., Vellinga, D., Ichikawa, N., Moon, C., Arikawa-Hirasawa, E., et al. (2007). Novel extracellular matrix structures in the neural stem cell niche capture the neurogenic factor fibroblast growth Factor 2 from the extracellular milieu. Stem Cells 25, 2146-2157. doi: 10.1634/stemcells.20070082

Kerever, A., Yamada, T., Suzuki, Y., Mercier, F., and Arikawa-Hirasawa, E. (2015). Fractone aging in the subventricular zone of the lateral ventricle. J. Chem. Neuroanat. 66-67, 52-60. doi: 10.1016/j.jchemneu.2015.06.001

Kerever, A., Nagahara, F., Keino-Masu, K., Masu, M., van Kuppevelt, T. H., Vivès, R. R., et al. (2021). Regulation of fractone heparan sulfate composition in young and aged subventricular zone neurogenic niches. Glycobiology cwab081. doi: 10.1093/glycob/cwab081

Kjell, J., Fischer-Sternjak, J., Thompson, A. J., Friess, C., Sticco, M. J., Salinas, F., et al. (2020). Defining the adult neural stem cell niche proteome identifies key regulators of adult neurogenesis. Cell Stem Cell 26, 277.e8-293.e8. doi: 10.1016/j.stem.2020.01.002

Kreuger, J., and Kjellén, L. (2012). Heparan sulfate biosynthesis. J. Histochem. Cytochem. 60, 898-907. doi: 10.1369/0022155412464972

Kriegstein, A., and Alvarez-Buylla, A. (2009). The glial nature of embryonic and adult neural stem cells. Annu. Rev. Neurosci. 32, 149-184. doi: 10.1146/annurev. neuro.051508.135600

Kriska, J., Honsa, P., Dzamba, D., Butenko, O., Kolenicova, D., Janeckova, L., et al. (2016). Manipulating Wnt signaling at different subcellular levels affects the fate of neonatal neural stem/progenitor cells. Brain Res. 1651, 73-87. doi: 10.1016/j.brainres.2016.09.026

Kurup, S., Wijnhoven, T. J. M., Jenniskens, G. J., Kimata, K., Habuchi, H., Li, J. P., et al. (2007). Characterization of anti-heparan sulfate phage display antibodies AO4B08 and HS4E4. J. Biol. Chem. 282, 21032-21042. doi: 10.1074/ jbc.M702073200

Lai, J., Chien, J., Staub, J., Avula, R., Greene, E. L., Matthews, T. A., et al. (2003). Loss of HSulf-1 up-regulates heparin-binding growth factor signaling in cancer. J. Biol. Chem. 278, 23107-23117. doi: 10.1074/jbc.M302203200

Lai, J., Chien, J. R., Moser, D. R., Staub, J. K., Aderca, I., Montoya, D. P., et al. (2004). hSulf1 sulfatase promotes apoptosis of hepatocellular cancer cells by decreasing heparin-binding growth factor signaling. Gastroenterology 126, 231-248. doi: 10.1053/j.gastro.2003.09.043

Lim, D. A., Tramontin, A. D., Trevejo, J. M., Herrera, D. G., García-Verdugo, J. M., and Alvarez-Buylla, A. (2000). Noggin antagonizes BMP signaling to create a niche for adult neurogenesis. Neuron 28, 713-726. doi: 10.1016/S0896-6273(00) 00148-3

Lois, C., and Alvarez-Buylla, A. (1994). Long-distance neuronal migration in the adult mammalian brain. Science 264, 1145-1148. doi: 10.1126/science.8178174

Luo, J., Daniels, S. B., Lennington, J. B., Notti, R. Q., and Conover, J. C. (2006). The aging neurogenic subventricular zone. Aging Cell 5, 139-152. doi: 10.1111/ j.1474-9726.2006.00197.x

Luo, J., Shook, B. A., Daniels, S. B., and Conover, J. C. (2008). Subventricular zone-mediated ependyma repair in the adult mammalian brain. J. Neurosci. 28, 3804-3813. doi: 10.1523/JNEUROSCI.0224-08.2008

Maslov, A. Y., Barone, T. A., Plunkett, R. J., and Pruitt, S. C. (2004). Neural stem cell detection, characterization, and age-related changes in the subventricular zone of mice. J. Neurosci. 24, 1726-1733. doi: 10.1523/JNEUROSCI.4608-03. 2004 
Mazzon, C., Anselmo, A., Cibella, J., Soldani, C., Destro, A., Kim, N., et al. (2011). The critical role of agrin in the hematopoietic stem cell niche. Blood 118, 2733-2742. doi: 10.1182/blood-2011-01-331272

Mercier, F. (2016). Fractones: extracellular matrix niche controlling stem cell fate and growth factor activity in the brain in health and disease. Cell. Mol. Life Sci. 73, 4661-4674. doi: 10.1007/s00018-016-2314-y

Mercier, F., Cho Kwon, Y., and Kodama, R. (2011). Meningeal/vascular alterations and loss of extracellular matrix in the neurogenic zone of adult BTBR $\mathrm{T}+\mathrm{tf} / \mathrm{J}$ mice, animal model for autism. Neurosci. Lett. 498, 173-178. doi: 10.1016/j. neulet.2011.05.014

Mercier, F., and Douet, V. (2014). Bone morphogenetic protein-4 inhibits adult neurogenesis and is regulated by fractone-associated heparan sulfates in the subventricular zone. J. Chem. Neuroanat. 5, 54-61. doi: 10.1016/j.jchemneu. 2014.03.005

Mercier, F., Kitasako, J. T., and Hatton, G. I. (2002). Anatomy of the brain neurogenic zones revisited: fractones and the fibroblast/macrophage network. J. Comp. Neurol. 451, 170-188. doi: 10.1002/cne.10342

Mercier, F., Kitasako, J. T., and Hatton, G. I. (2003). Fractones and other basal laminae in the hypothalamus. J. Comp. Neurol. 455, 324-340. doi: 10.1002/cne. 10496

Mercier, F., Kwon, Y. C., and Douet, V. (2012). Hippocampus/amygdala alterations, loss of heparan sulfates, fractones and ventricle wall reduction in adult BTBR T+tf/J mice, animal model for autism. Neurosci. Lett. 506, 208-213. doi: 10.1016/j.neulet.2011.11.007

Mohammadi, M., Olsen, S. K., and Ibrahimi, O. A. (2005). Structural basis for fibroblast growth factor receptor activation. Cytokine Growth Factor Rev. 16, 107-137. doi: 10.1016/j.cytogfr.2005.01.008

Narita, K., Chien, J., Mullany, S. A., Staub, J., Qian, X., Lingle, W. L., et al. (2007). Loss of HSulf-1 expression enhances autocrine signaling mediated by amphiregulin in breast cancer. J. Biol. Chem. 282, 14413-14420. doi: 10.1074/ jbc.M611395200

Nascimento, M. A., Sorokin, L., and Coelho-Sampaio, T. (2018). Fractone bulbs derive from ependymal cells and their laminin composition influence the stem cell niche in the subventricular zone. J. Neurosci. 38, 3880-3889. doi: 10.1523/ JNEUROSCI.3064-17.2018

Obermeier, B., Daneman, R., and Ransohoff, R. M. (2013). Development, maintenance and disruption of the blood-brain barrier. Nat. Med. 19, 15841596. doi: 10.1038/nm.3407

Pozzi, A., Yurchenco, P. D., and Iozzo, R. V. (2017). The nature and biology of basement membranes. Matrix Biol. 57-58, 1-11. doi: 10.1016/j.matbio.2016.12. 009

Pye, D. A., Vives, R. R., Turnbull, J. E., Hyde, P., and Gallagher, J. T. (1998). Heparan sulfate oligosaccharides require 6-O-sulfation for promotion of basic fibroblast growth factor mitogenic activity. J. Biol. Chem. 273, 22936-22942. doi: $10.1074 /$ jbc.273.36.22936

Ravikumar, M., Smith, R. A. A., Nurcombe, V., and Cool, S. M. (2020). Heparan sulfate proteoglycans: key mediators of stem cell function. Front. Cell Dev. Biol. 8:581213. doi: $10.3389 /$ fcell.2020.581213

Rojas-Vázquez, S., Blasco-Chamarro, L., López-Fabuel, I., Martínez-Máñez, R., and Fariñas, I. (2021). Vascular senescence: a potential bridge between physiological aging and neurogenic decline. Front. Neurosci. 15:666881. doi: 10.3389/fnins. 2021.666881

Sato, Y., Kiyozumi, D., Futaki, S., Nakano, I., Shimono, C., Kaneko, N., et al. (2019). Ventricular-subventricular zone fractones are speckled basement membranes that function as a neural stem cell niche. Mol. Biol. Cell 30, 56-68. doi: 10.1091/ mbc.E18-05-0286

Segarra, M., Aburto, M. R., and Acker-Palmer, A. (2021). Blood-brain barrier dynamics to maintain brain homeostasis. Trends Neurosci. 44, 393-405. doi: 10.1016/j.tins.2020.12.002

Seki, T., and Arai, Y. (1993). Highly polysialylated neural cell adhesion molecule (NCAM-H) is expressed by newly generated granule cells in the dentate gyrus of the adult rat. J. Neurosci. 13, 2351-2358. doi: 10.1523/jneurosci.13-06-02351. 1993
Shen, Q., Wang, Y., Kokovay, E., Lin, G., Chuang, S. M., Goderie, S. K., et al. (2008). Adult SVZ stem cells lie in a vascular niche: a quantitative analysis of niche cell-cell interactions. Cell Stem Cell 3, 289-300. doi: 10.1016/j.stem.2008.07. 026

Sugahara, K., and Kitagawa, H. (2002). Heparin and heparan sulfate biosynthesis. IUBMB Life 54, 163-175. doi: 10.1080/15216540214928

Sun, K. L. W., Correia, J. P., and Kennedy, T. E. (2011). Netrins: versatile extracellular cues with diverse functions. Development 138, 2153-2169. doi: 10.1242/dev.044529

Tavazoie, M., van der Veken, L., Silva-Vargas, V., Louissaint, M., Colonna, L., Zaidi, B., et al. (2008). A specialized vascular niche for adult neural stem cells. Cell Stem Cell 3, 279-288. doi: 10.1016/j.stem.2008.07.025

ten Dam, G. B., Hafmans, T., Veerkamp, J. H., and van Kuppevelt, T. H. (2003). Differential expression of heparan sulfate domains in rat spleen. J. Histochem. Cytochem. 51, 727-739. doi: 10.1177/00221554030510 0604

ten Dam, G. B., Kurup, S., van de Westerlo, E. M. A., Versteeg, E. M. M., Lindahl, U., Spillmann, D., et al. (2006). 3-O-sulfated oligosaccharide structures are recognized by anti-heparan sulfate antibody HS4C3. J. Biol. Chem. 281, 4654-4662. doi: 10.1074/jbc.M506357200

Thompson, S. M., Fernig, D. G., Jesudason, E. C., Losty, P. D., van de Westerlo, E. M. A., van Kuppevelt, T. H., et al. (2009). Heparan sulfate phage display antibodies identify distinct epitopes with complex binding characteristics: insights into protein binding specificities. J. Biol. Chem. 284, 35621-35631. doi: 10.1074/jbc.M109.009712

Uchimura, K., Morimoto-Tomita, M., Bistrup, A., Li, J., Lyon, M., Gallagher, J., et al. (2006). HSulf-2, an extracellular endoglucosamine-6-sulfatase, selectively mobilizes heparin-bound growth factors and chemokines: effects on VEGF. FGF-1, and SDF-1. BMC Biochem. 7:2. doi: 10.1186/14712091-7-2

van Kuppevelt, T. H., Dennissen, M. A. B. A., van Venrooij, W. J., Hoet, R. M. A., and Veerkamp, J. H. (1998). Generation and application of type-specific antiheparan sulfate antibodies using phage display technology. J. Biol. Chem. 273, 12960-12966. doi: 10.1074/jbc.273.21.12960

Viviano, B. L., Paine-Saunders, S., Gasiunas, N., Gallagher, J., and Saunders, S. (2004). Domain-specific modification of heparan sulfate by Qsulf1 modulates the binding of the bone morphogenetic protein antagonist noggin. J. Biol. Chem. 279, 5604-5611. doi: 10.1074/jbc.M310691200

Yamada, T., Kerever, A., Yoshimura, Y., Suzuki, Y., Nonaka, R., Higashi, K., et al. (2017). Heparan sulfate alterations in extracellular matrix structures and fibroblast growth factor-2 signaling impairment in the aged neurogenic niche. J. Neurochem. 142, 534-544. doi: 10.1111/jnc.14081

Yu, C., Griffiths, L. R., and Haupt, L. M. (2017). Exploiting heparan sulfate proteoglycans in human neurogenesis-controlling lineage specification and fate. Front. Integr. Neurosci. 11:28. doi: 10.3389/fnint.2017.00028

Conflict of Interest: The authors declare that the research was conducted in the absence of any commercial or financial relationships that could be construed as a potential conflict of interest.

Publisher's Note: All claims expressed in this article are solely those of the authors and do not necessarily represent those of their affiliated organizations, or those of the publisher, the editors and the reviewers. Any product that may be evaluated in this article, or claim that may be made by its manufacturer, is not guaranteed or endorsed by the publisher.

Copyright (c) 2021 Kerever and Arikawa-Hirasawa. This is an open-access article distributed under the terms of the Creative Commons Attribution License (CC BY). The use, distribution or reproduction in other forums is permitted, provided the original author(s) and the copyright owner(s) are credited and that the original publication in this journal is cited, in accordance with accepted academic practice. No use, distribution or reproduction is permitted which does not comply with these terms. 